\title{
Optimal Power Control and Scheduling for Energy Harvesting Wireless Networked Control Systems
}

\author{
Goksu Karadag \\ Electrical and Electronics Engineering \\ Koc University \\ Istanbul, Turkey \\ gkaradag16@ku.edu.tr
}

\author{
Sinem Coleri Ergen \\ Electrical and Electronics Engineering \\ Koc University \\ Istanbul, Turkey \\ sergen@ku.edu.tr
}

\begin{abstract}
In this paper, we introduce RF energy harvesting paradigm into WNCS framework to study the optimal power control, energy harvesting and scheduling problem with the objective of providing maximum level of adaptivity under strict timing and reliability requirements employing the constant rate transmission model. We formulate the problem as a Mixed Integer Linear Programming Problem (MILP). We show the power allocation can be separated from the scheduling and harvesting at optimality. Then, we introduce a heuristic algorithm for the scheduling problem, periodic list scheduling (PLS), inspired from list scheduling of jobs with sequence dependent setup times on identical machines. We then demonstrate via extensive simulations the superiority of the proposed algorithm in terms of closeness to the optimal, adaptivity and runtime.

Index Terms-Wireless networked control systems, adaptivity, power control, scheduling, RF energy harvesting
\end{abstract}

\section{INTRODUCTION}

Wireless networked control systems (WNCSs) are spatially distributed systems in which sensors, actuators, and controllers connect via a wireless network [1]. The usage of wireless communication in control systems result in flexible network architectures, and reduces installation and maintenance costs. Consequently, WNCSs have been finding a broad range of application areas such as mobile sensor networks, remote surgery, automated highway systems and unmanned aerial vehicles.

The work on WNCSs generally assume battery powered sensor nodes. In [2] and [3], the energy consumption of the sensor nodes is minimized while satisfying the performance requirements of the WNCS. However, these mostly ignore the dependency of energy consumption on the transmission power of the sensor nodes. Real time algorithms used for the scheduling of controller tasks on a processor, such as Earliest Deadline First (EDF) that assigns tasks upon release, have been utilized for the scheduling of periodic transmission of the sensor nodes in WNCSs [4]. However, none of these frameworks aim to provide adaptivity to retransmissions and additional messaging of the nodes. The more recent work on WNCSs address this issue and focus on maximizing adaptivity [4], [5]. Nonetheless, none of these studies incorporate energy harvesting.
Radio frequency (RF) energy harvesting networks (RFEHN) stand out as a plausible solution to limited lifetime of sensor nodes by providing sustainable power supply from radio signals with frequency range from $3 \mathrm{kHz}$ to $300 \mathrm{GHz}$ used as a medium to carry energy. The formulations for multi-user scheduling of single hop RF-EHNs [6] mostly aim at throughput fairness, throughput maximization or utility optimization under energy harvesting constraints. However, these formulations do not consider the requirements of control systems, such as the periodic nature of data transmissions, and are not honed to adapt to the changes in the network such as retransmissions, requirements of the sensors and network topology.

The goal of this paper is to study the optimal power control and scheduling algorithm that provides the maximum level of adaptivity while meeting the packet generation period, transmission delay, reliability and energy harvesting requirements of the sensor nodes in a WNCS.

\section{SYSTEM MODEL AND ASSUMPTIONS}

The system model and assumptions are detailed as follows.

1) The WNCS contains a number of sensor nodes. The nodes sample and transmit their sensed data either periodically (time-triggered) or upon triggering of an event (event-triggered) to a controller via wireless channel. The controller does not have multi-reception capability, i.e it can receive a packet from a single node at a time. Receiving the sampled information, controller computes and forwards a control command to the actuators as in Fig. 1-a. We assume a single-hop network with the controller having complete topology information, and responsible for scheduling and resource allocation of the active links.

2) We consider Time Division Multiple Access as MAC protocol [4]. Time is divided into fixed length frames which are further partitioned into a beacon and a number of packet slots, i.e. subframes. At the beginning of each subframe, the RF energy broadcast occurs, called energy harvesting slot, where the nodes that are scheduled to transmit within the corresponding subframe harvest energy. The controller uses the beacon to ensure time synchronization within the network and broadcast the 
scheduling and resource allocation decisions. At the end of transmissions, a beacon is transmitted to deliver the schedule for retransmissions as needed.

3) The nodes harvest energy from an RF resource dedicated to the controller. The nodes are equipped with a rechargable battery with low energy storage capacity and high discharge rate. Thus, all harvested energy is assumed to be consumed or discharged within the corresponding subframe [7].

4) The packet generation period of a sensor is either a multiple or an aliquot of other packet generation periods, which can be given as a constraint to the control applications [5].

5) We consider the energy consumption only during the data transmission as it is much larger than that in the sleep and transient modes [8].

6) We assume all devices in the network are equipped with a single omnidirectional antenna and operate over the same frequency.

7) The total energy harvested by a node from an RF source, denoted by $e_{i}$, is given as

$$
e_{i}=\zeta_{i} p_{c} g_{i i} t^{h},
$$

$\zeta_{i} \in(0,1)$ is the energy harvesting efficiency of node $i, p_{c}$ is the transmit power at the RF source, $g_{i i}$ is the channel gain of link $i$ which is assumed to be constant during the schedule, and $t^{h}$ is the length of the energy harvesting slot [9]. $\zeta_{i}=\zeta$ is assumed to be constant for all nodes in this paper.

8) We use the constant rate transmission model [10] in which each active link $i$ assigned a time slot transmits successfully at constant rate $r$ if link $i$ satisfies a fixed SINR treshold, given as

$$
\frac{p_{i} g_{i i}}{N_{0}} \geq \beta_{i}
$$

where $p_{i}$ is the transmit power of link $i, \beta_{i}$ is the SINR ratio to be kept by the link $i$ to achieve constant rate $r$, and $N_{0}$ is the background noise energy plus interference.

9) Fixed determinism is usually preferred over bounded determinism in control systems [11]. Therefore, we assume that given the packet generation period and transmission delay requirements $\left(T_{i}, d_{i}\right)$ provided for link $i$, the length of the time slot $t_{i}$, which is given by the ratio of the packet length of sensor $i, L_{i}$, to data rate $r$, is the same in all periods. The nodes have implicit delay tolerances, i.e $d_{i}=T_{i}$ for node $i$, which states that the nodes should complete transmission before the next data generation. The sensor records data samples at the beginning of the subframe every $T_{i}$ time unit, right before the energy harvesting slot. All nodes assigned for transmission within the corresponding subframe store the required energy for data transmission during the energy harvesting slot and send one data packet that consists of the data samples that it has recorded within the delay tolerance. No priority order is assumed among

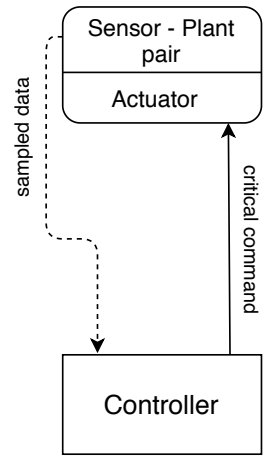

a)

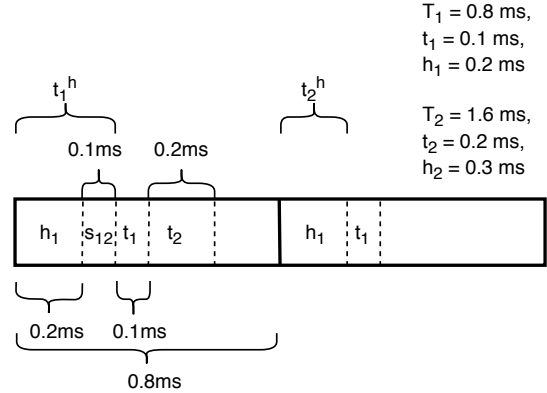

b)
Fig. 1: a) Simplified system architecture b) Sensor allocation example

the sensors. The packets of event-triggered sensors are transmitted in the unallocated parts of the schedule as they arrive.

\section{DESCRIPTION OF THE OPTIMIZATION PROBLEM}

\section{A. Objective Function}

The goal of the joint power control, energy harvesting and scheduling problem is to provide maximum adaptivity, which is a metric introduced in [4], and summarized as follows.

Adaptivity of a schedule is the ability of accommodating unscheduled changes such as a retransmission of a timetriggered, or transmission of an event-triggered sensor. It requires distribution of node transmissions as uniformly as possible over time units, i.e. the subframes [4]. total active length of a subframe $l$ is originally defined as the sum of the length of time slots allocated to subframe $l$ but extended here to additionally include the energy harvesting slot. The subframe and the frame lengths are fixed to the minimum and the maximum of the packet generation periods of all nodes in [4], denoted by $S$ and $F$, respectively. The objective is quantified as minimizing the maximum total active length of all $M$ subframes, where $M=F / S$.

\section{B. Constraints of the Problem}

The constraints of the optimization problem are the energy harvesting, transmission delay and the periodic data generation requirements of individual sensor nodes. The energy harvesting requirement necessitates that the energy harvesting slot allocated at the beginning of a subframe should suffice for the data transmission of all the sensors assigned to transmit in the corresponding subframe. The transmission delay requirement necessitates the generated packet to be transmitted before the next packet generation, i.e in $T_{i}$ time unit for node $i$. The periodic data generation requirement necessitates that the data generation of all sensors occur periodically.

Let $s_{i}$ be the ratio of packet generation period $T_{i}$ to the subframe length $S$. We now show that the periodic data generation and transmission delay requirements can be achieved 
by the allocation of fixed-length time slot once in every $s_{i}$ subframes.

Lemma 1: The periodic data generation and delay requirements are satisfied by allocating a time slot of length $t_{i}$ once in every $s_{i}$ subframes to node $i$, where $i \in[1, L]$.

Proof: Let the $k^{t h}$ data generation of sensor $i$ occur at the beginning of subframe $j$ at time $t_{g}^{(k)}=S(j-1)$. The $(k+1)^{s t}$ data generation will occur $T_{i}$ units after the $k^{\text {th }}$ data generation at time $t_{g}^{(k+1)}=S(j-1)+T_{i}=S(j-1)+S s_{i}=S(j-$ $\left.1+s_{i}\right)$ due to the periodic data generation requirement. Also, the $k_{t h}$ data transmission time, defined as $t_{t}^{(k)}$ must take place before $(k+1)^{t h}$ data generation, due to the delay requirement. Resultantly, we have $S(j-1) \leq t_{t}^{(k)} \leq S\left(j-1+s_{i}\right)$, which corresponds to allocating a time slot of length $t_{i}$ for node $i$ between subframes $j$ and $\left(j+s_{i}\right)$, i.e. once in every $s_{i}$ subframes.

\section{Formulation of Optimization Problem}

The optimal energy harvesting, scheduling and power control problem is mathematically formulated as follows:

minimize

$$
\max _{j \in[1, M]} \sum_{i=1}^{L} z_{i j} t_{i}+t_{j}^{h}
$$

subject to

$$
\begin{gathered}
\sum_{j=k s_{i}+1}^{(k+1) s_{i}} z_{i j}=1, \quad k \in\left[0, \frac{M}{s_{i}}-1\right], i \in[1, L] \\
\sum_{i=1}^{L} z_{i j} t_{i}+t_{j}^{h} \leq S, \quad j \in[1, M] \\
z_{i j} t_{i} p_{i} \leq \zeta_{i} p_{c} g_{i i} t_{j}^{h}, \quad i \in[1, L], j \in[1, M] \\
p_{i} \leq p_{\max }, \quad j \in[1, M] \\
\frac{p_{i} g_{i i}}{N_{0}} \geq \beta_{i}, \quad i \in[1, L]
\end{gathered}
$$

\section{variables}

$p_{i} \geq 0, z_{i j} \in\{0,1\}, t_{j}^{h} \geq 0, i \in[1, L], j \in[1, M]$

where $p_{\max }$ is the maximum allowed transmission power; $z_{i j}$ is an integer variable that takes value " 1 " if sensor $i$ is allocated to subframe $j$ and " 0 " otherwise, $t_{j}^{h}$ is the length of the energy harvesting slot of subframe $j$.

Eqn. (3a) aims to minimize the maximum total active length of all subframes. Eqns. (3b) and (3d) represent the periodic packet generation and delay requirements, and energy harvesting requirement, respectively. Eqn. (3c) is a feasibility condition that states that the maximum active length over all subframes cannot exceed the subframe length. Eqn. (3e) states that the transmission power of link $i$ cannot exceed maximum allowed transmit power. Eqn. (3f) represents SINR required to achieve constant rate in link $l$ with no concurrent transmissions. The variables of the problem are given as follows: 1) $z_{i j}, i \in[1, L], j \in[1, M]$ for scheduling; 2) $p_{i}, i \in[1, L]$ for power allocation; and 3) $t_{j}^{h}, j \in[1, M]$ for energy harvesting slot.
We now show that optimal power allocation can be separated from the optimal scheduling and enery harvesting problem, and simplify the energy harvesting constraints.

\section{Analysis of the Formulation}

1) Optimal Power Allocation: From Eqns. (3d) and (3f), we can obtain $\zeta p_{c} g_{i i} t_{j}^{h} \geq z_{i j} t_{i} p_{i} \geq z_{i j} t_{i}\left(\frac{\left.\beta_{i} N_{0}\right)}{g_{i i}}\right.$ for $i \in$ $[1, L], j \in[1, M]$. Expanding $t_{i}$, we can replace Eqn. (3d) in the formulation by Eqn. (4) since Eqn. (3d) can easily be obtained from Eqn. (4) and vice versa, knowing Eqn. (3f), where:

$$
\zeta p_{c} g_{i i} t_{j}^{h} \geq z_{i j}\left(\frac{L_{i}}{r}\right)\left(\frac{\beta_{i} N_{0}}{g_{i i}}\right)
$$

By this reduction, the only equations in the formulation involving $p_{i}$ are Eqns. (3e) and (3f). Since $\frac{\beta_{i} N_{0}}{g_{i i}} \leq p_{\max }$ has to hold for any feasible solution, the optimal power is the lower bound that achieves the required SINR, i.e.

$$
p_{i}^{*}=\frac{\beta_{i} N_{0}}{g_{i i}}, \quad i \in[1, L]
$$

2) Energy Harvesting Slot Allocation: From Eqn. (4), we extract $t_{j}^{h}$ as:

$$
t_{j}^{h} \geq \frac{z_{i j}\left(\frac{L_{i}}{r}\right)\left(\frac{\beta_{i} N_{0}}{g_{i i}}\right)}{\zeta p_{c} g_{i i}}
$$

Let us define $h_{i}$ as the energy harvesting time required for the transmission of link $i$. Obviously, from Eqn. (6), $h_{i}=$ $\frac{\left(\frac{L_{i}}{r}\right)\left(\frac{\beta_{i} N_{0}}{g_{i i}}\right)}{\zeta p_{c} g_{i i}}$ for $i \in[1, L]$. Let us also define the sets $I_{j}$ and $H_{j}, I_{j} g_{i i}=\left\{i \mid z_{i j}=1\right\}$ and $H_{j}=\left\{h_{i} \mid i \in I_{j}\right\}$, for $j \in[1, M]$. In other words, $I_{j}$ is the index set of sensors assigned to subframe $j$, and $H_{j}$ is the set of the energy harvesting time values of the sensors assigned to subframe $j$. In any feasible solution, we have $t_{j}^{h} \geq h_{i}, i \in I_{j}$.

In the optimal solution, we have $t_{j}^{h *} \in H_{j}, t_{j}^{h *}=\max _{i \in I_{j}} h_{i}$ for $j \in[1, M]$.

3) Simplified Model: The simplified formulation may be written as follows:

\section{minimize}

$$
W
$$

subject to

$$
\begin{gathered}
\sum_{j=k s_{i}+1}^{(k+1) s_{i}} z_{i j}=1, \quad k \in\left[0, \frac{M}{s_{i}}-1\right], i \in[1, L] \\
t_{j}^{h} \geq z_{i j} h_{i}, \quad i \in[1, L], j \in[1, M] \\
S \geq W \geq \sum_{i=1}^{L} z_{i j}\left(\frac{L_{i}}{r}\right)+t_{j}^{h}, \quad j \in[1, M]
\end{gathered}
$$

variables

$W \geq 0, z_{i j} \in\{0,1\}, t_{j}^{h} \in H_{j}, i \in[1, L], j \in[1, M]$

Eqn. (7a) is used to transform the objective from a nonlinear form of minimizing $\max _{x} f(x)$ to a linear form and merged with Eqn. (3c) to obtain Eqn. (7d). The variables of the 
problem are $z_{i j}, i \in[1, L], j \in[1, M]$, continuous variable $W$, and $t_{j}^{h} \in H_{j}, j \in[1, M]$. The resultant problem is MILP.

Because the problem is MILP, which is generally NP-hard, we will propose a polynomial-time heuristic algorithm.

\section{Periodic List Scheduling (PLS) Algorithm}

In this section, we will propose a heuristic for the scheduling problem formulated in Section III-D3 based on a heuristic proposed for minimum makespan scheduling (MSP) on identical machines. To propose our heuristic algorithm, we will adopt a concept existent in machine scheduling problems, called sequence-dependent setup times, or shortly, setup times. In machine scheduling problems, sequence-dependent setup time $s_{i j}$ is the required setup time of a machine when task $j$ is to be executed immediately following task $i$. The list scheduling algorithm proposed for the MSP on parallel identical machines with sequence-dependent setup times schedules the next job on a given list to the next available machine for processing [12]. The setup times are considered by incorporating their values in processing times [13]. In other words, the modified processing time of a task $j$ following task $i$ on a machine is given by the sum of its processing time $t_{j}$ and the setup time $s_{i j}$. Similarly, in our scheduling framework, the processing time of a task $j$ on subframe $k$ depends on the other nodes that have been scheduled on subframe $k$ priorly. The processing time of a node is its time slot length $t_{j}$ plus the additional harvesting time it requires, which depends on the other nodes that have been scheduled priorly on subframe $k . s_{i j}$ corresponds to the additional harvesting requirement of sensor $j$ with respect to sensor $i$. If sensor $j$ is assigned to subframe $k$ where sensor $i$ has already been assigned, sensor $j$ will require additional $s_{i j}$ time units of energy harvesting upon assignment. In other words, sensor $j$ requires $s_{i j}=\max \left(0, h_{j}-h_{i}\right)$ units of additional harvesting time when it is to be allocated to subframe $k$ where sensor $i$ has been previously allocated. Then, processing time of sensor $j$ on subframe $k$ is given by $p_{j}^{k}=t_{j}+\min _{i \in I_{k}} s_{i j} . s_{j j}, j \in[1, L]$ is defined to be the inherent energy harvesting requirement of sensor $j$, and consequently set to $h_{j}$. If the subframe is empty so far, then sensor $j$ will require $s_{j j}=h_{j}$ units of harvesting time. In the following, we will propose the scheduling algorithm PLS that assigns sensors to the subframe with the smallest total active length.

The workings of the algorithm PLS can be found in Algorithm 1 . At the initialization, the nodes in the list are ordered in increasing packet generation periods (Line 2). Then, all additionally required harvesting times of nodes with respect to one another, $s_{i j}$, are calculated (Line 4). After calculation, the next node $j$ on the list is assigned to the subframe with the minimum total active length with processing time $p_{j}^{k}$ (Line 6). Finally, the time slot and $s_{i j}$ assignment is repeated every $s_{j}$ subframes for $j \in[1, L]$, satisfying 1 (Line 7). A simple visual for the allocation of two nodes is given in Fig. 1-b.

\section{PERFORMANCE EVALUATION}

The goal of this section is to evaluate the performance of the proposed scheduling algorithm PLS compared to the optimal

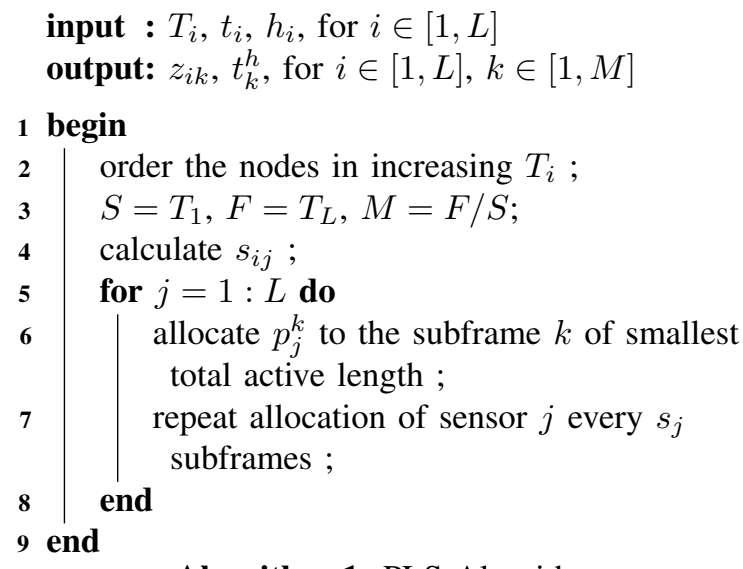

Algorithm 1: PLS Algorithm

TABLE I: Simulation Parameters

\begin{tabular}{||c|c||c|c||c|c||}
\hline$\alpha$ & 3.5 & $\sigma_{z}$ & $4 \mathrm{~dB}$ & $\beta_{i}, \forall i$ & $10 \mathrm{~dB}$ \\
\hline$N_{0}$ & $10^{-8} \mathrm{~W} / \mathrm{Hz}$ & $f$ & $915 \mathrm{MHz}$ & $d_{0}$ & $1 \mathrm{~m}$ \\
\hline$\zeta$ & 0.7 & $p_{c}$ & $4 \mathrm{~W}$ & $r$ & $2 \mathrm{Mbps}$ \\
\hline
\end{tabular}

algorithm and the commonly used Earliest Deadline First algorithm in terms of maximum total active length; maximum aperiodic delay, which is the worst-case delay that an aperiodic packet experiences until transmission in the unallocated part of the schedule [4]; and average runtime. The optimal algorithm is obtained by choosing the smallest amongst the evaluations of all feasible solutions. EDF algorithm [14] is a real-time scheduling algorithm that assigns priorities to tasks inversely proportional to their deadlines. In our framework, we consider the non-preemptive counterpart of EDF [15] for fair comparison, where a running task is uninterrupted until completion.

Simulation results are obtained based on 100 independent random network topologies. The attenuation of the links are determined by considering both large and small-scale statistics. The attenuation of the links considering the large scalestatistics is modeled as

$$
P L_{d B}(d)=P L_{d B}\left(d_{0}\right)+10 \alpha \log _{10}\left(d / d_{0}\right)+Z
$$

where $d$ is the distance between the sensor node and the controller, $P L(d)$ is the path loss at distance $d, P L\left(d_{0}\right)$ is the free space path loss at reference distance $d_{0}, \alpha$ is the path loss exponent and $Z$ is a Gaussian random variable with zero mean and standard deviation $\sigma_{z}$. The small scale fading has been modeled as Rayleigh fading with scale parameter $\Omega$ set to the mean power level determined by Eqn. (8) [4]. The distances between sensor nodes and controller are randomly distributed in the range $[0.1,1] \mathrm{m}$. The packet generation periods and the packet lengths are uniformly chosen from the set $\{1,5,10,50,100,200\} \mathrm{ms}$ and the interval [10,20] bytes, respectively. All simulations are performed in MATLAB where IBM CPLEX solver is used for the optimal algorithm. The simulation parameters are based on the parameters of existing 


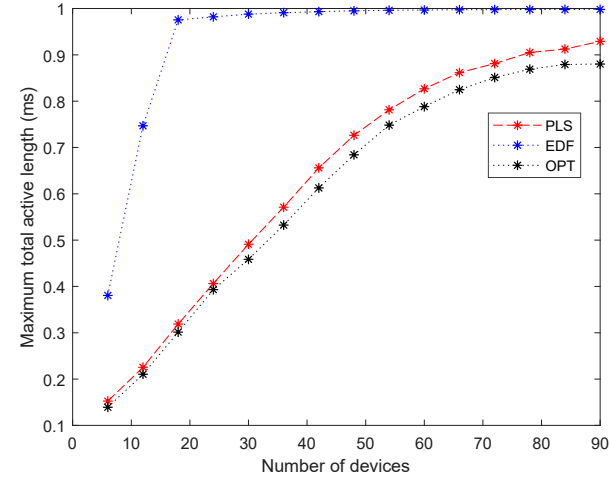

Fig. 2: Maximum active length vs Number of devices for PLS, EDF, OPT algorithms.

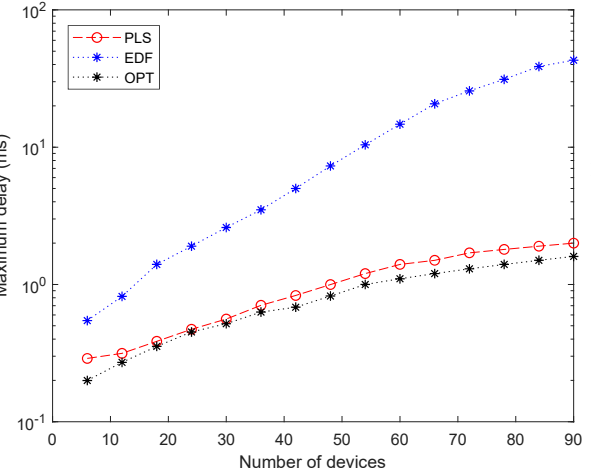

Fig. 3: Maximum delay vs Number of devices for PLS, EDF, OPT algorithms.

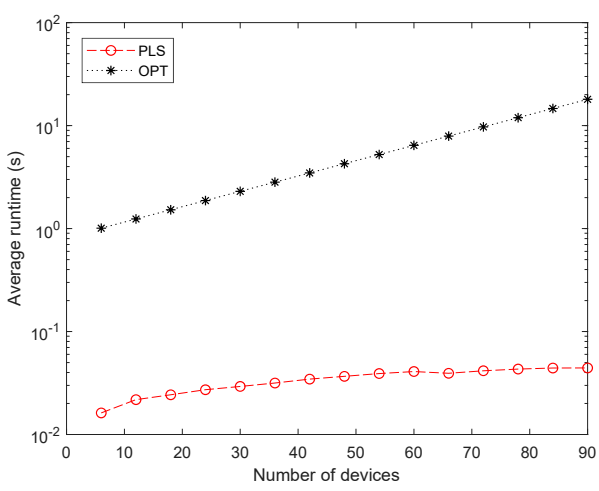

Fig. 4: Average runtime vs Number of devices for PLS, OPT algorithms.
RF transmitter implementations, such as the Powercaster [16], as listed in Table I.

Fig. 2 shows the maximum total active length of the subframes for different numbers of nodes and different scheduling algorithms, including PLS, EDF and the optimal solution. The performances of PLS is very close to the optimal solution with maximum approximation ratio of 1.0556. PLS outperforms EDF since EDF schedules tasks as they arrive.

Fig. 3 shows the performance of the scheduling algorithms in terms of maximum delay experienced by an aperiodic packet. PLS outperforms EDF with a close-to-optimal performance. For EDF, tasks are scheduled as they arrive, some of the subframes have almost no room for the allocation of additional packets. As the number of nodes increases, the number of fully allocated subframes that the additional packets have to wait to transmit increases. On the other hand, PLS distributes the allocations as uniformly as possible over the subframes, allowing the allocation of additional messaging.

Fig. 4 shows the average runtime of the algorithms. The average runtime of PLS is negligible when compared to that of the optimal, which exponentially increases as the number of nodes increases.

\section{CONCLUSION}

In this paper, we study the optimal power control and scheduling with the aim of providing maximum level of adaptivity to accommodate the changes in transmission time, retransmissions, and allocation of additional messages while satisfying the packet generation period, transmission delay, reliability and energy harvesting requirements of the sensor nodes for WNCSs. The optimal power allocation is separated from the scheduling and energy harvesting problem. The resultant problem is MILP, for which we propose a polynomial time scheduling heuristic, PLS, based on identical machine scheduling algorithms with sequence-dependent setup times. Finally, through simulations, we illustrate the very close-tooptimal performance and superiority of the proposed heuristic compared to commonly used EDF and optimal algorithm in terms of adaptivity and average runtime for various network sizes.

\section{REFERENCES}

[1] J. P. Hespanha, P. Naghshtabrizi, and Y. Xu, "A survey of recent results in networked control systems," Proceedings of the IEEE, vol. 95, no. 1, pp. 138-162, 2007.

[2] P. G. Park, J. Araújo, and K. H. Johansson, "Wireless networked control system co-design." in ICNSC, 2011, pp. 486-491.

[3] J. Araújo, A. Anta, M. Mazo Jr, J. Faria, A. Hernandez, P. Tabuada, and K. H. Johansson, "Self-triggered control over wireless sensor and actuator networks," in International Conference on Distributed Computing in Sensor Systems and Workshops (DCOSS), 2011. IEEE, 2011, pp. $1-9$.

[4] Y. Sadi and S. C. Ergen, "Optimal power control, rate adaptation and scheduling for uwb-based intra-vehicular wireless sensor networks," IEEE Trans. Vehicular Technology, vol. 62, no. 1, pp. 219-234, 2013.

[5] — "Energy and delay constrained maximum adaptive schedule for wireless networked control systems," IEEE Transactions on Wireless Communications, vol. 14, no. 7, pp. 3738-3751, 2015.

[6] X. Lu, P. Wang, D. Niyato, D. I. Kim, and Z. Han, "Wireless networks with rf energy harvesting: A contemporary survey," IEEE Communications Surveys \& Tutorials, vol. 17, no. 2, pp. 757-789, 2015.

[7] Z. Hadzi-Velkov, I. Nikoloska, H. Chingoska, and N. Zlatanov, "Opportunistic scheduling in wireless powered communication networks," IEEE Transactions on Wireless Communications, vol. 16, no. 6, pp. 41064119, 2017.

[8] S. Cui, A. J. Goldsmith, and A. Bahai, "Energy-constrained modulation optimization," IEEE transactions on wireless communications, vol. 4, no. 5, pp. 2349-2360, 2005.

[9] H. Ju and R. Zhang, "Throughput maximization in wireless powered communication networks," IEEE Transactions on Wireless Communications, vol. 13, no. 1, pp. 418-428, 2014.

[10] Y. Sadi and S. C. Ergen, "Minimum length scheduling with packet traffic demands in wireless ad hoc networks," IEEE Transactions on Wireless Communications, vol. 13, no. 7, pp. 3738-3751, 2014.

[11] J. R. Moyne and D. M. Tilbury, "The emergence of industrial control networks for manufacturing control, diagnostics, and safety data," Proceedings of the IEEE, vol. 95, no. 1, pp. 29-47, 2007.

[12] I. M. Ovacik and R. Uzhoy, "Worst-case error bounds for parallel machine scheduling problems with bounded sequence-dependent setup times," Operations Research Letters, vol. 14, no. 5, pp. 251-256, 1993.

[13] M. Kurz and R. Askin, "Heuristic scheduling of parallel machines with sequence-dependent set-up times," International journal of production research, vol. 39, no. 16, pp. 3747-3769, 2001.

[14] F. Liu, A. Narayanan, and Q. Bai, "Real-time systems," 2000.

[15] N. Guan, W. Yi, Z. Gu, Q. Deng, and G. Yu, "New schedulability test conditions for non-preemptive scheduling on multiprocessor platforms," in 2008 Real-Time Systems Symposium. IEEE, 2008, pp. 137-146.

[16] Powercast. ([Online]). [Online]. Available: http://www.powercastco. $\mathrm{com} /$ 